\title{
The New Morris Method: an efficient second order screening method
}

\author{
Roger A. Cropp*, Roger D. Braddock \\ Faculty of Environmental Sciences, Griffith University, Nathan Campus, 4111, Queensland, Australia.
}

* Corresponding author

\begin{abstract}
The New Morris Method was proposed by Campolongo and Braddock [1] as an extension of the Morris Method [2] to include estimation of two-factor interaction effects. An undetected programming error prevented Campolongo and Braddock from appreciating the efficacy of the method. Testing on an analytic function reveals that the method is more powerful and efficient than previously thought.
\end{abstract}

Keywords: sensitivity analysis, New Morris Method, two-factor interactions, computational efficiency

\section{Introduction}

Sensitivity analysis is a fundamental tool in the building, use and understanding of mathematical models. In many areas of research, parameter values are poorly known and/or difficult to measure [3]. Sensitivity analysis can identify the most important parameters in a model, and indicate the 'robustness' of a model.

The New Morris Method [1] utilised a graph theory solution to the 'handcuffed prisoner' problem [4,5] to devise the most efficient way of sampling a parameter space to provide the minimal number of model evaluations required to be able to calculate two-factor interaction effects (second-order effects) in addition to the first- 
order effects estimated by the Morris Method. The reader is directed to Campolongo and Braddock [1] for a full description of the method. Subsequent testing of the original code provided by Campolongo and Braddock revealed an error in the code. This affected the calculation of both first and second-order effects. This paper corrects and extends the results obtained by Campolongo and Braddock, and in doing so reveals the true efficacy of the New Morris Method.

\section{Theoretical background}

Consider a function

$$
f=f(w),
$$

where $w$ is a vector of parameters $\left(0 \leq w_{i} \leq 1\right)$ defined on the unit cube $R$. The firstorder effects of variations in the parameter values $w_{i}$ on the function $f$ are given by:

$$
E E_{i}=\frac{d f}{d w_{i}}
$$

and the second-order effects of interactions between parameter values $w_{i}$ and $w_{j}$ are given by:

$$
T F E_{i j}=\frac{d^{2} f}{d w_{i} d w_{j}} .
$$

The first-order sensitivity of equation (1) to variations in $w_{i}$ is estimated by the Morris Method as the average $\left[\mu_{i}(f)\right]$ of all the $E E_{i}$ 's, where the average is taken over samples in the parameter space. The sampling method is discussed by Morris [2]. The exact sensitivity to first-order effects $\left[\mathrm{M}_{i}(f)\right]$ may be obtained analytically:

$$
\mathrm{M}_{i}(f)=\frac{1}{V} \int_{R} E E_{i} d V,
$$


where the integral is taken over the parameter volume $R$. Morris also used the standard deviation $\left[\sigma_{i}(f)\right]$ of the $E E_{i}$ 's as an indicator of the presence of higher order effects. Likewise, this may be obtained analytically:

$$
\Sigma_{i}(f)=\frac{1}{\sqrt{V}} \sqrt{\int_{R}\left(E E_{i}-\mathrm{M}_{i}(f)\right)^{2} d V} .
$$

The global second-order sensitivity of equation (1) to interactions of variations in $w_{i}$ and $w_{j}$ is estimated by the New Morris Method as the average $\left[\lambda_{i j}(f)\right]$ of all the $T F E_{i j}$ 's, where the average is again taken over samples in $R$. The 'handcuffed prisoner' sampling method is discussed in [1]. The exact sensitivity to second-order effects $\left[\Lambda_{i j}(f)\right]$ may be obtained analytically:

$$
\Lambda_{i j}(f)=\frac{1}{V} \int_{R} T F E_{i j} d V
$$

We shall demonstrate that the standard deviations of the second-order effects $\left[\rho_{i j}(f)\right]$ may be used as an indication of the existence of higher-order effects in the New Morris Method. These may also be calculated analytically:

$$
\mathrm{P}_{i j}(f)=\frac{1}{\sqrt{V}} \sqrt{\int_{R}\left(T F E_{i j}-\Lambda_{i j}(f)\right)^{2} d V} .
$$

Campolongo and Braddock used an analytic function containing first and secondorder effects to test the New Morris Method. We shall repeat these tests, but also extend them and include analytic expressions for $\mathrm{M}_{i}(f), \Sigma_{i}(f), \Lambda_{i j}(f)$ and $P_{i j}(f)$, to elucidate the accuracy of the method. All functions are defined on the fourdimensional unit cube $R$, and include coefficient values defined for functions with lower-order effects.

We will initially examine a function $\left(f_{1}\right)$ containing only first-order effects: 


$$
f_{1}=\sum_{i=1}^{4} b_{i} w_{i}
$$

where:

$$
b_{i}=\left[\begin{array}{c}
0.05 \\
0.59 \\
10 \\
0.21
\end{array}\right]
$$

For this function, the first-order effects $\left[\mathrm{M}_{i}\left(f_{1}\right)\right]$ are just the coefficients $b_{i}$ :

$$
\mathbf{M}_{i}\left(f_{1}\right)=b_{i}
$$

As there are no second-order effects, the Morris method should derive these values exactly, with zero standard deviations $\left[\sigma_{i}\left(f_{l}\right)\right]$ of the means. Campolongo and Braddock used an analytic function containing first and second-order effects, which we will denote $f_{2}$, to test the New Morris Method

$$
f_{2}=\sum_{i=1}^{4} b_{i} w_{i}+\sum_{i \leq j}^{4} b_{i j} w_{i} w_{j}
$$

where

$$
b_{i j}=\left[\begin{array}{cccc}
0 & 80 & 60 & 40 \\
0 & 30 & 0.73 & 0.18 \\
0 & 0 & 0.64 & 0.93 \\
0 & 0 & 0 & 0.06
\end{array}\right] .
$$

The exact first-order effects for this function are given by:

$$
\mathbf{M}_{i}\left(f_{2}\right)=\left[\begin{array}{l}
b_{1}+b_{11}+\frac{1}{2} b_{12}+\frac{1}{2} b_{13}+\frac{1}{2} b_{14} \\
b_{2}+b_{22}+\frac{1}{2} b_{12}+\frac{1}{2} b_{23}+\frac{1}{2} b_{24} \\
b_{3}+b_{33}+\frac{1}{2} b_{13}+\frac{1}{2} b_{23}+\frac{1}{2} b_{34} \\
b_{4}+b_{44}+\frac{1}{2} b_{14}+\frac{1}{2} b_{24}+\frac{1}{2} b_{34}
\end{array}\right]=\left[\begin{array}{l}
90.050 \\
71.045 \\
41.470 \\
20.825
\end{array}\right]
$$

The standard deviations $\left[\sigma_{i}\left(f_{2}\right)\right]$ of the first-order effects for this function may be calculated as: 


$$
\Sigma_{i}\left(f_{2}\right)=\left[\begin{array}{l}
\left(b_{1}-\mathbf{M}_{1}\left(f_{2}\right)\right)\left(b_{1}+2 b_{11}+b_{12}+b_{13}+b_{14}-\mathbf{M}_{1}\left(f_{2}\right)\right) \\
+b_{11}\left(\frac{4}{3} b_{11}+b_{12}+b_{13}+b_{14}\right)+b_{12}\left(\frac{1}{3} b_{12}+\frac{1}{2} b_{13}+\frac{1}{2} b_{14}\right) \\
+b_{13}\left(\frac{1}{3} b_{12}+\frac{1}{2} b_{14}\right)+\frac{1}{3} b_{14}^{2} \\
\left(b_{2}-\mathbf{M}_{2}\left(f_{2}\right)\right)\left(b_{2}+b_{12}+2 b_{22}+b_{23}+b_{24}-\mathbf{M}_{2}\left(f_{2}\right)\right) \\
+b_{22}\left(b_{12}+\frac{4}{3} b_{22}+b_{23}+b_{24}\right)+b_{23}\left(\frac{1}{2} b_{12}+\frac{1}{3} b_{23}+\frac{1}{2} b_{24}\right) \\
+b_{24}\left(\frac{1}{2} b_{12}+\frac{1}{3} b_{24}\right)+\frac{1}{3} b_{12}^{2} \\
\left(b_{3}-\mathbf{M}_{3}\left(f_{2}\right)\right)\left(b_{3}+b_{13}+b_{23}+2 b_{33}+b_{34}-\mathbf{M}_{3}\left(f_{2}\right)\right) \\
+b_{33}\left(b_{13}+b_{23}+\frac{4}{3} b_{33}+b_{34}\right)+b_{34}\left(\frac{1}{2} b_{13}+\frac{1}{2} b_{23}+\frac{1}{3} b_{34}\right) \\
+b_{13}\left(\frac{1}{3} b_{13}+\frac{1}{2} b_{23}\right)+\frac{1}{3} b_{23}^{2} \\
\left(b_{4}-\mathbf{M}_{1}\left(f_{2}\right)\right)\left(b_{4}+b_{14}+b_{24}+b_{34}+2 b_{44}-\mathbf{M}_{4}\left(f_{2}\right)\right) \\
+b_{44}\left(b_{14}+b_{24}+b_{34}+\frac{4}{3} b_{44}\right)+b_{14}\left(\frac{1}{3} b_{14}+\frac{1}{2} b_{24}+\frac{1}{2} b_{34}\right) \\
+b_{24}\left(\frac{1}{3} b_{24}+\frac{1}{2} b_{34}\right)+\frac{1}{3} b_{34}^{2} \\
=\left[\begin{array}{l}
31.08 \\
28.86 \\
17.75 \\
11.54
\end{array}\right] .
\end{array}\right.
$$

The New Morris Method will not find the first-order effects $\left[\mu_{i}\left(f_{2}\right)\right.$ and $\left.\sigma_{i}\left(f_{2}\right)\right]$ precisely due to the presence of second-order effects, which confound the estimates of the first-order effects. The exact second order effects $\left[\Lambda_{i j}\left(f_{2)}\right)\right.$ may be calculated from equation (11), and are given by the off-diagonal elements of $b_{i j}$ :

$$
\Lambda_{i j}\left(f_{2}\right)=\left[\begin{array}{cccc}
0 & 80 & 60 & 40 \\
0 & 0 & 0.73 & 0.18 \\
0 & 0 & 0 & 0.93 \\
0 & 0 & 0 & 0
\end{array}\right] \text {. }
$$

Again, as there are no higher-order effects in this model, we can expect the New Morris Method to find the second-order effects $\left[\lambda_{i j}\left(f_{2}\right)\right]$ exactly, with zero standard deviations $\left[\rho_{i j}\left(f_{2}\right)\right]$. 
Finally, we shall consider an extension of equation (11) to include third-order interactions:

$$
f_{3}=\sum_{i=1}^{4} b_{i} w_{i}+\sum_{i \leq j}^{4} b_{i j} w_{i} w_{j}+\sum_{i \leq j \leq k}^{4} b_{i j k} w_{i} w_{j} w_{k}
$$

To simplify the analysis of the full model incorporating third-order effects (equation (16)), we shall consider each second-order interaction (ij) with only one third-order interaction. This allows us to reduce the three-dimensional matrix $b_{i j k}$ to a twodimensional matrix $b_{i j 4}$, to which we assign the following values:

$$
b_{i j 4}=\left[\begin{array}{cccc}
0 & 10 & 0.98 & 0.19 \\
0 & 0 & 0.49 & 50 \\
0 & 0 & 0 & 1 \\
0 & 0 & 0 & 0
\end{array}\right] .
$$

The exact first-order effects are now given by:

$$
\begin{aligned}
\mathbf{M}_{i}\left(f_{3}\right) & =\left[\begin{array}{c}
b_{1}+b_{11}+\frac{1}{2} b_{12}+\frac{1}{2} b_{13}+\frac{1}{2} b_{14}+\frac{1}{4} b_{124}+\frac{1}{4} b_{134}+\frac{1}{3} b_{144} \\
b_{2}+b_{22}+\frac{1}{2} b_{12}+\frac{1}{2} b_{23}+\frac{1}{2} b_{24}+\frac{1}{4} b_{124}+\frac{1}{4} b_{234}+\frac{1}{3} b_{244} \\
b_{3}+b_{33}+\frac{1}{2} b_{13}+\frac{1}{2} b_{23}+\frac{1}{2} b_{34}+\frac{1}{4} b_{134}+\frac{1}{4} b_{234}+\frac{1}{3} b_{344} \\
b_{4}+b_{44}+\frac{1}{2} b_{14}+\frac{1}{2} b_{24}+\frac{1}{2} b_{34}+\frac{1}{4} b_{124}+\frac{1}{4} b_{134}+\frac{1}{4} b_{234}+\frac{1}{2} b_{144}+\frac{1}{2} b_{244}+\frac{1}{2} b_{344}
\end{array}\right] \\
& =\left[\begin{array}{c}
92.855 \\
90.338 \\
42.171 \\
49.283
\end{array}\right],
\end{aligned}
$$

and the exact second-order effects are given by:

$$
\begin{aligned}
\Lambda_{i j}\left(f_{3}\right) & =\left[\begin{array}{cccc}
0 & b_{12}+\frac{1}{2} b_{124} & b_{13}+\frac{1}{2} b_{134} & b_{14}+\frac{1}{2} b_{124}+\frac{1}{2} b_{134}+b_{144} \\
0 & 0 & b_{23}+\frac{1}{2} b_{234} & b_{24}+\frac{1}{2} b_{124}+\frac{1}{2} b_{234}+b_{244} \\
0 & 0 & 0 & b_{34}+\frac{1}{2} b_{134}+\frac{1}{2} b_{234}+b_{344} \\
0 & 0 & 0 & 0
\end{array}\right] \\
& =\left[\begin{array}{cccc}
0 & 85 & 60.49 & 45.68 \\
0 & 0 & 0.975 & 55.42 \\
0 & 0 & 0 & 2.27 \\
0 & 0 & 0 & 0
\end{array}\right] .
\end{aligned}
$$


The indicator of second and higher-order interactions $\left[\Sigma_{i}\left(f_{3}\right)\right]$ is now given by very messy expressions, so the expected values will only be given for these thus:

$$
\Sigma_{i}\left(f_{3}\right)=\left[\begin{array}{l}
32.89 \\
39.71 \\
16.84 \\
26.82
\end{array}\right] .
$$

The indicator of third- and higher-order effects $\left[P_{i j}\left(f_{3}\right)\right]$ will be given by:

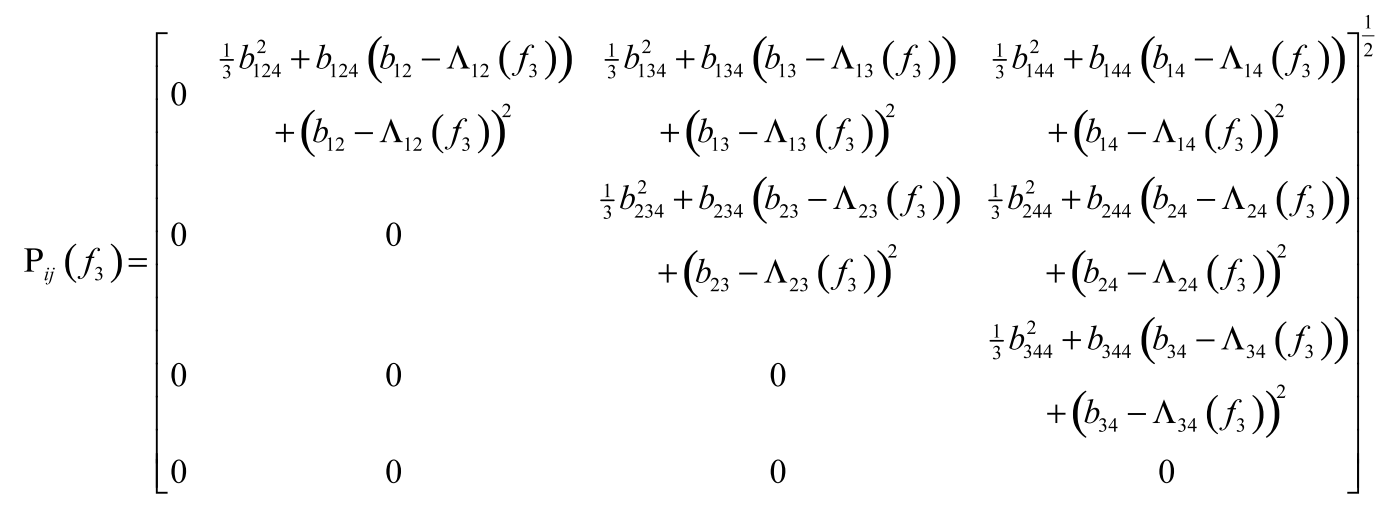

$$
=\left[\begin{array}{cccc}
0 & 2.89 & 0.28 & 5.58 \\
0 & 0 & 0.14 & 24.3 \\
0 & 0 & 0 & 0.88 \\
0 & 0 & 0 & 0
\end{array}\right] .
$$

The presence of the third-order effects can be expected to preclude the New Morris Method from finding all lower order effects, $\mu_{i}\left(f_{2}\right), \sigma_{i}\left(f_{2}\right), \lambda_{i j}\left(f_{3}\right)$ and $\rho_{i j}\left(f_{3}\right)$, exactly.

\section{Experimental testing}

The Morris and New Morris Methods require the user to set two parameters, $r$ and $\Delta$. The range of each factor is divided into segments of size $\Delta$, the resolution at which the factor is examined, and the method is repeated in a number of runs $(r)$, with the estimates of the effects calculated using combined data from all runs. In our initial 
investigations, we will use the values of $r$ and $\Delta$ as used by Campolongo and Braddock, and will address optimising these parameters later.

\section{Results}

Table 1 reveals that in the absence of higher order effects, the New Morris Method is able to estimate the first-order effects with great accuracy and efficiency, returning precise estimates of both $\mu_{i}\left(f_{1}\right)$ and $\sigma_{i}\left(f_{1}\right)$. The addition of second order effects (Table 2) reduces the accuracy with which the method is able to estimate $\mu_{i}\left(f_{2}\right)$ and $\sigma_{i}\left(f_{2}\right)$, but it is able to estimate the higher-order effects exactly. The method indicates that the model contains at most second-order effects by estimating $\lambda_{i j}\left(f_{2}\right)$ and $\rho_{i j}\left(f_{2}\right)$ precisely, with $\rho_{i j}\left(f_{2}\right)=0$ confirming that there are no third or higher-order effects.

The addition of third-order effects to the model (Table 3) again reduces the accuracy of the estimate of $\mu_{i}\left(f_{3}\right)$ and $\sigma_{i}\left(f_{3}\right)$, with larger values for $\sigma_{i}\left(f_{3}\right)$ correctly indicating that more influential higher-order effects are present than in the second-order model. Likewise, the existence of third-order effects degrades the accuracy of the estimate of $\lambda_{i j}\left(f_{3}\right)$, with non-zero values estimated for $\rho_{i j}\left(f_{3}\right)$ revealing the presence of higher-order interactions. In this case, with no higher-order effects than third-order, the method is able to accurately reflect the relative magnitudes of the third-order effects prescribed by $b_{i j 4}$.

\section{Discussion}


The progressive degradation of estimates for first-order effects as higher-order interactions are added to the model, evidenced in Tables $1-3$, is counter-intuitive. Each path used in the New Morris Method (see [1] for details of paths) provides two estimates of each first-order effect and one estimate of each second-order interaction. The Central Limit Theorem suggests that we should therefore expect the first-order effects to be more accurately estimated than the second-order effects. This is clearly not the case and is likely the result of the influence of higher-order interactions on the first-order effects. This suggests that some care should be taken in determining the number of runs and the resolution to be used in the New Morris Method.

Figure 1 illustrates the mean relative error of the New Morris Method's estimate of $\mu_{i}\left(f_{3}\right)$ in relation to the number of runs and the resolution used in the method. Figure 1 clearly shows that there is no benefit in increasing the number of runs used if the resolution is very low, although only a small improvement in the resolution is required to obtain accurate results.

Figure 1 also reveals that if the resolution is reasonable, the Morris Method requires only a few runs to be able to estimate $\mu_{i}\left(f_{3}\right)$ quite accurately. For resolutions $(\Delta)$ of approximately 5 or greater, the estimates will be within about $10 \%$ of the true value provided the number of runs $(r)$ is also more than about five. Doubling the number of runs to ten halves the error to about $5 \%$, but increases in the number of runs and/or the resolution from this level appear to offer diminishing returns. Increasing $r$ and $\Delta$ to 100 only reduces the error to about $3 \%$. It is therefore questionable whether the extra computation is justified, especially in cases where the model is expensive to evaluate. It is likely, however, that optimal $r$ and $\Delta$ will vary with different models. 
The errors in the estimation of $\lambda_{i j}\left(f_{3}\right)$ (Figure 2) while similar form to those of $\mu_{i}\left(f_{3}\right)$, are approximately half the magnitude at low resolutions and runs, confirming the implications of Tables $1-3$ that despite using less data to estimate the mean, the second-order effects are generally calculated more accurately than the first-order effects. Similarly to the first-order effects, little is to be gained by increasing $r$ and $\Delta$ above approximately 10 for this model. The first and second-order effects are both estimated with similar accuracy (about 3\%) for large numbers of runs at high resolution.

Estimates of effects using very few runs (Figures 1 and 2) give highly variable results, with the mean error of the estimates of up to $35 \%$. No consistent improvement in accuracy can be gained by increasing the resolution, although a single run can deliver estimates with errors of as little as $5 \%$. The variation in accuracy at low run numbers appears to be random, therefore offering little hope of increasing efficiency by selecting a suitable resolution.

Our investigations have revealed that while the New Morris Method can accurately estimate the highest-order effects in a model, the existence of large interactions of more than two factors prevents the method from precisely estimating first or secondorder effects. In these cases, relatively few runs of the method at moderate resolution are sufficient to provide accurate estimates of $\mu_{i}(f)$ and $\lambda_{i j}(f)$. The number of runs is more critical to obtaining a good estimate of the effects than the resolution at which the parameter space is searched, provided both are not very small. 


\section{Summary and conclusions}

We have examined the New Morris Method after correcting an error in the computer program used by Campolongo and Braddock [1] to initially demonstrate the method. Our investigations have revealed that the method is able to precisely and accurately estimate first-order effects in models containing at most first-order factor interactions, and second-order effects in models containing at most second-order factor interactions. It can also provide accurate indications of first-order effects in models containing second and higher-order effects, and of second-order effects in models containing third and higher-order effects.

We have further demonstrated that the New Morris Method can also provide indications that third and/or higher-order interactions are present in models by returning non-zero standard deviations for the means of the second-order effects. While the method is not able to resolve these effects, the relative magnitudes of the standard deviations appear a reliable guide to the relative importance of each factor interaction.

Finally, our tests have demonstrated that analyses using small numbers of runs at quite coarse resolutions can provide very good estimates of the sensitivity of a model to parameter perturbations, and interactions of up to three parameters. Indeed, there is little improvement in the accuracy of the method to be gained by increasing the number of runs, or the resolution at which the factors are sampled, above quite small values. This confirms the New Morris Method as a powerful and efficient method for global sensitivity analysis. 


\section{References}

[1] Campolongo F, Braddock RD. The use of graph theory in the sensitivity analysis of the model output: a second order screening method. Reliability Engineering and System Safety, 1999; 64: 1-12.

[2] Morris MD. Factorial sampling plans for preliminary computational experiments. Technometrics, 1991; 33(2): 161-174.

[3] Fennel K, Losch M, Schroter J, Wenzel M. Testing a marine ecosystem model: sensitivity analysis and parameter optimisation. Journal of Marine Systems. 2001; 28: 45-63.

[4] Hell P, Rosa A. Graph decomposition, handcuffed prisoners and balanced pdesigns. Discrete Mathematics, 1972; 2: 229-252.

[5] Hung SHY, Mendelshon NS. Handcuffed designs. Discrete Mathematics, 1977; 18: 23-33.

\section{Figure captions}

Figure 1: Mean relative error magnitude of first-order effects as a function of the number of runs and the resolution of the New Morris Method (errors are averages of three implementations of the method). 
Figure 2: Mean relative error magnitude of second-order effects as a function of the number of runs and the resolution of the New Morris Method (errors are averages of three implementations of the method). 